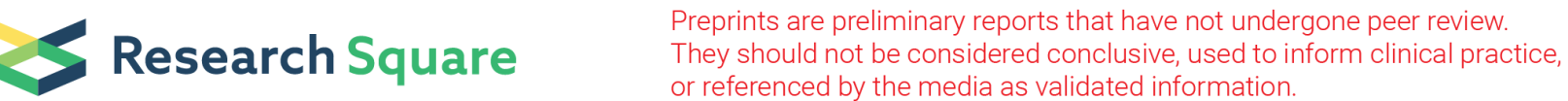

\section{Fluoroscopy-assisted percutaneous guide wire puncture for the treatment of intertrochanteric fractures with proximal femur nail anti-rotation}

Huazhong Xu

the Affiliated Wuxi No:2 People's Hospital of Nanjing Medical University https://orcid.org/0000-00017869-9307

\section{Peng Yuan}

the Affiliated Wuxi People's Hospital of Nanjing Medical University

\section{Yusheng Yang}

the Affiliated Wuxi No:2 People's Hospital of Nanjing Medical University

Keqin Shi ( $\nabla 13771523579 @ 163 . c 0 m)$

The Affiliated Wuxi No.2 People's Hospital of Nanjing Medical University https://orcid.org/0000-00024144-5597

\section{Research article}

Keywords: intertrochanteric fracture, Proximal femoral nail anti-rotation, (PFNA), percutaneous puncture, fixation

Posted Date: July 29th, 2020

DOI: https://doi.org/10.21203/rs.3.rs-47414/v1

License: (c) (i) This work is licensed under a Creative Commons Attribution 4.0 International License. Read Full License 


\section{Abstract}

Background: Accurate percutaneous guide wire insertion for the treatment of intertrochanteric fractures with proximal femur nail anti-rotation(PFNA) can be a technical challenge. It is critical for the surgeon to place the implant accurately during the procedure; however, the muscles and soft tissues in the hip often make accurate guide wire insertion difficult, especially in overweight and obese patients.

Purposes: The purpose of this study was to evaluate a series of patients who underwent percutaneous guide wire puncture and PFNA fixation to determine the influence of this technique on soft-tissue trauma and accuracy of guide wire placement.

Methods: From June 2015 to June 2018,forty-five consecutive patients with a minimally displaced or displaced intertrochanteric fracture were treated with percutaneous guide wire puncture technique and PFNA fixation in two trauma centers. Six patients were lost to follow-up. The remaining thirty-nine patients were followed until union or a minimum of twelve months. There were thirty female and fifteen male patients with a mean age of 78.5 years old. Radiographs were used to evaluate the quality of fracture union. Intraoperative fluoroscopic views of the first puncture attempt were evaluated for the accuracy of percutaneous puncture technique.The number of puncture attempts used was recorded.Incision lengths of nail insertion were measured and recorded.

Results: All fractures united. The length of the incisions ranged from $2.0 \mathrm{~cm}$ to $3.1 \mathrm{~cm}$, with a mean of $2.5 \mathrm{~cm}$. The angles between the guide wire and the axis of the proximal femur were within $3^{\circ}$ in lateral fluoroscopic view,and ranged from $4^{\circ}$ to $8^{\circ}$ with a mean value of $6.3^{\circ}$ in anteroposterior(AP) fluoroscopic views. The number of punctures in every patient were less than 2.Among forty-five patients, forty-three patients(95.6\%) were punctured successfully with one pass. No complications were observed.

Conclusions: Percutaneous guide wire puncture for the treatment of intertrochanteric fractures with PFNA fixation can lead to excellent outcomes, by virtue of accurate implant placement, less trauma,and fewer complications.

Level of Evidence: Therapeutic level IV

\section{Introduction}

Fractures of the intertrochanteric region of the femur are one of the most common fractures encountered by orthopedic surgeons[1].PFNA has been accepted as one standard treatment of choice for intertrochanteric fractures $[2,3,4]$ because of its biomechanical superiority compared with plates $[5,5,7,8$, $9,10,11,12,13,14,15]$.However, the muscles and soft tissues in the hip,especially in overweight and obese patients,make accurate percutaneous guide wire insertion for nail fixation difficult[16].The accurate guide wire insertion is critical for accurate nail placement.In this study,fluoroscopy was used to guide the insertion of a 2.8-mm-diameter guide wire percutaneously and a 5.5-mm-diameter cannulated drill was used to create a 5.5-mm-diameter bone canal accurately in order to facilitate insertion of another 2.8-mm- 
diamter guide wire through a small incision. The use of the percutaneous puncture technique allows the surgeon to position the guide wire accurately without the blockage from surrounding anatomy.This greatly ensures accurate implant placement.

\section{Materials And Methods}

From June 2015 to June 2018, 45 consecutive patients with minimally displaced or displaced intertrochanteric fractures were treated using the percutaneous guide wire puncture technique and PFNA fixation in two trauma centers. Fractures with an Orthopedic Trauma Association[17]classification of $31 \mathrm{~A} 1$ and 31A2 were included. Fractures for which anatomical reduction could not be achieved by closed manipulation were excluded from this study. The hospital's institutional review board approval and patient consent were obtained for this study. Patients were followed until clinical and radiographic union had occurred or for a minimum of 12 months.Mean follow-up time was 15 months and ranged from 12 to 24 months.Six patients were lost to follow-up.Of the remaining thirty-nine patients,twenty-five were female and fourteen were male.The mean age was 78.5 years with a range of 46 to 100 years. Radiographs were used to evaluate fracture union. The lengths of the incision used for nail insertion were measured and recorded. After guide wires were inserted, the angles between the axis of the proximal femur and the guide wire in AP and lateral fluoroscopic views were measured and recorded and used to assess the accuracy of the percutaneous puncture technique.

\section{Surgical Technique}

All operations were performed under spinal anesthesia.Patients were positioned supine on a radiolucent fracture table with the fractured limb placed in boot traction and the contralateral limb placed in the hemilithotomy position. The $\mathrm{C}$-arm machine was placed between limbs. All fractures were successfully reduced by closed manipulation under fluoroscopy before beginning the operation. Prophylactic antibiotics,usually a first generation cephalosporin,were given.

The first step was to determine the projection of the tip of the greater trochanter in the skin and the insertion direction of the guide wire in the coronal plane. Under lateral fluoroscopy,a 2-mm-diameter kirschner wire was attached to the lateral skin of the hip(Fig. 1A).The position of the kirschner wire was adjusted to locate its image in the central portion of the medullary cavity of the proximal femur(Fig. 1B). A marked line was made along the kirschner wire in the skin. We named it as line A.Then the C-arm was rotated 90 degrees to obtain a AP fluoroscopic view of the proximal femur.Under AP fluoroscopy,two 2mm-diameter kirschner wires were placed in the skin over the anterior part of the hip(Fig. 2A). Under AP fluoroscopic guidance,the first kirschner wire was placed transversely and kept perpendicular to the longitudinal axis of the femur. When its image ran through the tip of the greater trochanter(Fig. 2B),a line was drawn along the kirschner wire on the anterior skin and a vertical line(Line B)was drawn on the lateral skin of the hip.In the meantime, another kirschner wire was placed longitudinally in the skin. Under AP fluoroscopy guidance, its position was adjusted to make its image run through the tip of the greater trochanter and the central point of the medullary cavity of the area that is $1 \mathrm{~cm}$ from the inferior edge of 
the lesser trochanter, or its image was located in the surgeon's desired location(Fig. 2C). Then, the second Kirschner wire was attached to the skin. Another 2-mm-diameter kirschiner wire with a suture tied in its tip was suspended and contacted the second kirschner wire and the lateral skin of the thigh(Fig. 3). The contact point in the lateral skin was also marked. Another vertical line(Line $C)$ that ran through this marked point was drawn in the lateral skin,which represented the projected direction of the X-rays beam under AP fluoroscopy. The two vertical lines crossed with line A in the lateral skin. The distal crossing point(Point a)was the projection of the tip of the greater trochanter in the lateral skin, and the proximal crossing point(Point b)was the entry point of the guide wire in the skin(Fig. 4).

The second step was to insert the guide wire percutaneously under fluoroscopic control. A $0.5 \mathrm{~cm}$-lenghth incision was made at the entry point of the guide wire. Using line A as a guide, a 2.8-mm-diameter guide wire was inserted into soft tissues through the stab incision to touch the tip of the greater trochanter(Fig. 5A). Under AP and lateral fluoroscopy, the insertion direction of the guide wire in both coronal and sagittal plane were adjusted to ensure the tip of the guide wire was located in the tip of the greater trochanter(Fig. 5B-C).A hammer was used to position the guide wire into the bone.Under AP fluoroscopy, the guide wire should run through the central point of the medullary cavity, which is about $1 \mathrm{~cm}$ away from the inferior edge of the lesser trochanter of the femur or the angle between the axis of the proximal femur and guide wire should be about $5^{\circ}$ (Fig. 6A). Under lateral fluoroscopy,the the guide wire should be located in the central part of the medullary cavity of the proximal femur and kept parallel to the axis of the proximal femur(Fig. 6B). Otherwise, the insertion direction or the entry point of the guide wire in the tip of the greater trochanter was adjusted. Then a 5.5-mm-diameter cannulated drill (the cannulated drill was usually used to create a bone canal in femoral neck to place 7.3-mm-diameter cannulated screws in the treatment of femoral neck fractures) was inserted into the soft tissues through the stab incision over the 2.8-mm-diameter guide wire to contact the tip of the greater trochanter and drilled into the bone to about $6 \mathrm{~cm}$ in depth(Fig. 7A-B). A 5.5-mm-diameter bone canal was made by the cannulated drill. Under AP and lateral fluoroscopy, the bone canal obtains a correct entry point in the greater trochanter and an accurate insertion direction in both the sagittal and coronal planes,which was desired by the operating surgeon.

The third step was to use a long 2.8-mm-diameter guide wire to replace the short 2.8-mm-diameter guide wire through an approximately $2-\mathrm{cm}$ incision located in line A.From about $4 \mathrm{~cm}$ away from the distal crossing point in line A, one $2.5-\mathrm{cm}$ incision was made to contact the guide wire. The muscle was stripped along the guide wire until the tip of the greater trochanter was contacted.Then the 2.8-mm-diameter guide wire was removed from the bone canal,and a long guide wire was inserted into the bone canal through the approximately $2.5-\mathrm{cm}$ incision until distal part of the medullary cavity of the femur(Fig. 8A-B).

The remaining steps were the same as conventional procedure. After incision closure,the length of the incision was measured and recorded.(Fig. 9A-D).

\section{Results}


All fractures united. No wound-related, nerve-related or vascular-related complications were observed. The lengths of the nail insertion incisions ranged from $2.0 \mathrm{~cm}$ to $3.1 \mathrm{~cm}$, with a mean of $2.5 \mathrm{~cm}$. The angle between the guide wire and the axis of the proximal femur in lateral fluoroscopic views were within $3^{\circ}$ and ranged from $0-3^{\circ}$, with a mean of $0.8^{\circ}$. The mean value of the angle between the guide wire and the axis of the proximal femur in AP fluoroscopic views was $6.3^{\circ}$ and ranged from $4^{\circ}$ to $8^{\circ}$. The mean number of guide wire puncture times was 1.04 times and ranged from 1 to 2 times.95.6\% patients were punctured successfully with one pass, and no other adjustment was required.Two puncture attempts were required in two patients.(Table.1)

\section{Discussion}

Percutaneous puncture technique has been applied in minimally invasive spine surgery widely[18,19,20,21,22,]since it was reported in vertebral-body biopsy in 1956[23].It can lead to excellent clinical outcomes because it is less invasive and more precise. In 1997, Anthony Young used a percutaneous puncture technique to insert a guide wire into the intervertebral foramen and completed his first percutaneous endoscopic lumbar discectomy(PELD) procedure[22].Thomas HoogLand reported that he used the same percutaneous puncture technique to create a bone canal in the lumbar upper articular process and finished his percutaneous endoscopic lumbar discectomy (PELD) procedure[20, 21].In 1987, Galibert and Deramond applied a percutaneous puncture technique to creation of double bone canals in both pedicles, and completed the percutaneous vertebroplasty(PVP) procedure[18].Actually,when percutaneous puncture technique was applied in trauma surgery, it can provide the same excellent clinical outcomes. In 1989,Matta and Saucedo reported the treatment of pelvic ring fractures using percutaneous puncture technique in their study[24].By creating a soft-tissue tunnel and a bone canal,the percutaneous puncture technique can make accurate implant placement easy and quick,which is significant to fracture union and reduction of soft-tissue trauma.

PFNA fixation has been accepted as one standard treatment of choice for intertrochanteric fractures,especially for unstable and displaced fractures[2, 3, 4].Compared with dynamic hip system(DHS) and proximal femur locked plate(PFLP), the PFNA has more biomechanical superiorities[5, $6,7,8,9,10,11,12,13,14,15]$.Its design was based on the anatomic characters of fracture zones. For adults, the PFNA was designed with $5^{\circ}$ valgus angle in order to facilitate nail insertion through a small incision. There was a crossing point between the axis of the proximal part of the nail and the distal part. When the PFNA was inserted into the medullary cavity of the proximal femur in an accurate manner, the crossing point of two axes was typically located in the central point of the medullary cavity of the area, which was about $1 \mathrm{~cm}$ away from the inferior edge of the lesser trochanter(Fig. 6A). The guide wire should run through the tip of greater trochanter and the central point of the medullary cavity of this area(Fig. 6A). Under AP fluoroscopy, the two points can be visualized to determine the insertion direction of the guide wire in the coronal plane,and the entry point of the guide wire in the skin.

The percutaneous puncture techniques for minimally invasive surgery need accurate navigation equipment.To determine the entry points of the guide wire in the skin and in the bone of femur, the $\mathrm{C}$-arm 
machine was the most commonly used equipment. It is essential for the surgeon to understand the characters of fluoroscopy imaging. The "vector effect" and "plane effect" of the x-rays beams emitted from the x-rays tube are two important characters that can be used to determine entry point and insertion direction of the guide wire. Under AP or lateral fluoroscopy, the image of the guide wire on the screen represents not only a linear shadow, but also one plane. This plane was formed by the guide wire and the projected direction of the X-rays beams emitted from the tube.Under AP fluoroscopy,the projected direction of X-rays is perpendicular to the ground,which can be demonstrated by a hanging Kirschner wire.Two kirschner wires formed one plane(Fig. 3).This is the "vector effect" and "plane effect" of the fluoroscopy imaging during the procedure. When the vertical plane crossed the horizontal plane,the crossed line of the two planes is the puncturing route of the guide wire in the muscles and soft tissues of the hip. The crossing point of the crossed line with the lateral skin is the entry point of the guide wire(Fig. 4). The marked lines of the two planes in the skin can also be used to guide the insertion of the guide wire. When inserting the guide wire through the stab incision in line $A$, it is important to ensure that the horizontal projection of the guide wire coincides with line A(Fig. 5A-C).In this study,the accuracy of the first puncture attempt was $95.6 \%$.Among the forty-five patients,forty-three patients were punctured successfully with one pass, and the remaining two required two puncture attempts. In the first puncture attempts of these two patients,although the guide wire was parallel to the axis of the proximal femur in lateral fluoroscopy view, their positions were not located in the central part of the medullary cavity of the proximal femur.Adjustment of the entry point of the guide wire in the tip of the greater trochanter was required. This phenomenon can be explained by the principle of "image drifting" during fluoroscopy imaging,which was caused by the"scattering and amplification effect" of X-rays beams. By adjusting the image of the guide wire be located in the central part of the screen, the accuracy of the location of guide wire can be improved. Therefore,the percutaneous puncture technique based on fluoroscopy imaging is accurate,effective,and reproducible.

In the conventional procedure,the muscles and soft tissues in the hip often obstruct and prevent accurate insertion of the guide wire through a small incision,especially in overweight and obese patients. In order to improve the accuracy of guide wire insertion, it is necessary to abduct the fractured limb more or enlarge the incision to facilitate accurate guide wire insertion. However, if a bone canal with accurate entry point and insertion direction was predrilled(Fig. 7A-B), it is easy to inserted the guide wire into the bone canal through a small incision(Fig. 8A-B).In this study,the percutaneous puncture technique improved the accuracy of surgical operation without increasing soft-tissue trauma.

Accurate implant placement(Fig. 10A-D,Fig. 11A-C)is important for maintaining the reduction,providing excellent support,and achieving good biomechanical stability during fracture healing $[6,9,15]$.During operation,the bone canal was made by the drill over the guide wire.If the guide wires were inserted inaccurately,the nail would be placed in a wrong bone canal.Even if anatomic reduction was achieved,the nail insertion would result in displacement or fractures of the proximal or distal fragment.Under AP and lateral fluoroscopic views,the accuracy of the percutaneous puncture technique can be evaluated by observing the angles between the guide wire and the axis of the proximal femur.If the technique is accurate,the guide wire in lateral fluoroscopic view should be parallel to the axis of the proximal femur 
and located in the central portion of the medullary cavity(Fig. 6B).In this study,the angles in lateral fluoroscopic views were within $3^{\circ}$. Under AP fluoroscopic view, the angle between the guide wire and the axis of the proximal femur ranged from $4^{\circ}$ to $8^{\circ}$, with a mean of $6.3^{\circ}$, which is close to the $5^{\circ}$ valgus angle of PFNA(Fig. 6A).It has demonstrated that percutaneous puncture technique is accurate enough in determining the entry point and insertion direction of the guide wire.

With the evolution of the percutaneous puncture technique in the orthopedic trauma field,this technique may be applied in more minimally invasive surgeries,such as pelvic,spine,and acetabular percutaneous surgeries,especially for overweight and obese patients.

However,there are still several weaknesses in this study. The first is that we did not set a comparison group with the conventional surgery,and the second is that the number of patients is few. The third is that because the fracture is often associated with dispalcement of lesser trochanter,it is difficult to determine accurate position, which is $1 \mathrm{~cm}$ from the inferior edge of the lesser trochanter.In this condition,it is necessary for the surgeon to assess the $5^{\circ} \mathrm{valgus}$ angle between the guide wire with the axis of the proximal femur under AP fluoroscopic view.

\section{Conclusion}

Accurate percutaneous guide wire puncture for the treatment of intertrochanteric fractures with PFNA fixation can lead to excellent clinical outcomes with smaller incisions,minimally additional soft-tissue disruption, and a low rate of associated complications. It is a safe,precise,reproducible and minimally invasive technique.

\section{Abbreviations}

PFNA:Proximal Femoral Nail Anti-rotation; AP:Anteroposterior;

\section{Declarations}

\section{Availablility of data and materials}

All data generated and analysed during this study are included in this article.

\section{Ethics approval and consents to participate}

All procedures performed in studies involving human participants were in accordance with the ethical standards of the ethical committee of the affiliated wuxi No:2 people's hospital of Nanjing Medical University and with the 1964 Helsinki Declaration and its later amendments or comparable ethical standards.Informed consent was obtained from all individual participants included in the study. 


\section{Consent to publication}

Not applicable.

\section{Competing interests}

The authors declare that they have no competing interests.

\section{Disclosure}

The authors did not receive any outside funding or grants in support of their research for or preparation of this work. Neither of their immediate family received, in any one year, payments or other benefits or a commitment to provide sunch benefits from a commercial entity. No commercial entity paid or directed, or granted to pay or direct, any benefits to any research fund, foundation, division, center, clinical practice, or other charitable or nonprofit organization woth which the anthors, or a member of the immediate families, are affiliated or associated.

\section{Funding}

Not applicable.

\section{Authors' contribution}

Dr Huazhong $\mathrm{Xu}$ and Dr Ke-qin Shi designed this study and were responsible for the performing of all procedures.The manuscript was wrote by Dr Huazhong Xu. Dr Pen Yuan and Dr Yusheng Yang were responsible for the data collection and analysis.Dr Huazhong Xu was responsible for the follow-up of all patients.All authors read and approved the final manuscript.

\section{Acknowledgement}

We thank all the anonymous reviewers for their helpful suggestions on the quality improvement of this papeAvailabler.

\section{References}

1. Ehmke LW, Fitzpatrick DC, Krieg JC, Madey SM, Bottlang M. Lag screws for hip fracture fixation: evaluation of migration resistance under simulated walking[J]. Journal of Orthopaedic Research, 2005, 23(6): 1329-1335. 
2. Adams Cl. Robinson C M, McQueen MM. Prospective randomized controlled trial of an intramedullary nail versus dynamic screw and plate for intertrochanteric fractures of the femur[J]. J Orthop Trauma, 2001, 15(6): 394-400.

3. Egol KA. Capriccioso C E, Konda SR. Tejwani, N. C., Liporace, F. A., Zuckerman, J. D., \& Davidovitch, R. I.Cost-effective trauma implant selection: AAOS exhibit selection[J]. JBJS, 2014, 96(22): e189.

4. Socci AR, Casemyr NE, Leslie MP, Baumgaertner MR. Implant options for the treatment of intertrochanteric fractures of the hip: rationale, evidence, and recommendations[J]. The bone \& joint journal, 2017, 99(1): 128-133.

5. Celebi L. Can M, Muratli HH. Yagrmurlu MF,Yuksel HY,Bicimoglu A.Indirect reduction and biological internal fixation of comminuted subtrochanteric fractures of the femur[J]. Injury, 2006, 37(8): 74050.(5).

6. Haynes RC, Pöll RG, Miles AW, Weston RB. Failure of femoral head fixation: a cadaveric analysis of lag screw cut-out with the gamma locking nail and AO dynamic hip screw[J]. Injury, 1997, 28(5-6): 337-341.

7. Knobe M, Drescher W, Heussen N, Sellei RM, Pape HC. Is helical blade nailing superior to locked minimally invasive plating in unstable pertrochanteric fractures?[J]. Clinical Orthopaedics and Related Research $B, 2012,470(8)$ : 2302-2312.

8. Kung AWC. The Working Group for Formulating Clinical Management. Guidelines for Osteoporosis in Hong Kong[J]. Hong Kong Medical Journal, 1999.

9. Kuzyk PR, Lobo J, Whelan D, Zdero R, McKee MD, Schemitsch EH. Biomechanical evaluation of extramedullary versus intramedullary fixation for reverse obliquity intertrochanteric fractures[J]. Journal of orthopaedic trauma, 2009, 23(1): 31-38.

10. Matre K, Havelin LI, Gjertsen JE, Espehaug B, Fevang JM. Intramedullary nails result in more reoperations than sliding hip screws in two-part intertrochanteric fractures[J]. Clinical Orthopaedics and Related Research $\AA$, 2013, 471(4): 1379-1386.

11. Ricci WM, Schwappach J, Tucker M, Coupe K, Brandt A, Sanders R, Leighton R. Trochanteric versus piriformis entry portal for the treatment of femoral shaft fractures[J]. Journal of orthopaedic trauma, 2006, 20(10): 663-667.

12. Robinson CM. Houshian S, Khan LAK. Trochanteric-entry long cephalomedullary nailing of subtrochanteric fractures caused by low-energy trauma[J]. JBJS, 2005, 87(10): 2217-26.

13. Shukla S, Johnston P, Ahmad MA, Wynn-Jones H, Patel AD. \& Walton, N. P. Outcome of traumatic subtrochanteric femoral fractures fixed using cephalo-medullary nails[J]. Injury, 2007, 38(11): 12861293.

14. Starr AJ, Hay MT, Reinert CM, Borer DS, Christensen KC. Cephalomedullary nails in the treatment of high-energy proximal femur fractures in young patients: a prospective, randomized comparison of trochanteric versus piriformis fossa entry portal[J]. Journal of orthopaedic trauma, 2006, 20(4): 240246. 
15. Strauss E, Frank J, Lee J, Kummer FJ, Tejwani N. Helical blade versus sliding hip screw for treatment of unstable intertrochanteric hip fractures: a biomechanical evaluation[J]. Injury, 2006, 37(10): 984989.

16. Ostrum RF. Marcantonio A, Marburger R. A critical analysis of the eccentric starting point for trochanteric intramedullary femoral nailing[J]. J Orthop Trauma, 2005, 19(10): 681-6.

17. Meinberg EG, Agel J, Roberts CS, Karam MD, Kellam JF. Fracture and dislocation classification compendium-2018[J]. Journal of Orthopaedic trauma, 2018, 32: S1-S10.

18. Galibert P, Deramond H, Rosat P, Le DG. Preliminary note on the treatment of vertebral angioma by percutaneous acrylic vertebroplasty[J]. Neuro-chirurgie, 1987, 33(2): 166-168.

19. Hijikata S. Percutaneous nucleotomy. A new concept technique and 12 years' experience[J]. Clinical orthopaedics and related research, 1989 (238): 9-23.

20. Hoogland T, Schubert M, Miklitz B, Ramirez A. Transforaminal posterolateral endoscopic discectomy with or without the combination of a low-dose chymopapain: a prospective randomized study in 280 consecutive cases[J]. Spine, 2006, 31(24): E890-E897.

21. Schubert M. Hoogland T. Endoscopic transforaminal nucleotomy with foraminoplasty for lumbar disk herniation[J]. Operative Orthopadie Traumatologie, 2005, 17(6): 641-61.

22. Tsou PM. Yeung A T. Transforaminal endoscopic decompression for radiculopathy secondary to intracanal noncontained lumbar disc herniations: outcome and technique[J]. The Spine Journal, 2002, 2(1): 41-8.

23. Craig FS. Vertebral-body biopsy[J]. JBJS. 1956;38(1):93-102.

24. Matta JM. Saucedo T. Internal fixation of pelvic ring fractures[J]. Clinical orthopaedics and related research, 1989 (242): 83-97.

\section{Tables}

Table.1凶Clinical data and results 


\begin{tabular}{|c|c|c|c|c|c|c|c|}
\hline Number & Gender & $\begin{array}{l}\text { Age } \\
\text { \yr囚 }\end{array}$ & Left/Right & $\begin{array}{l}\text { Puncturing } \\
\text { times }\end{array}$ & $\begin{array}{l}\text { Length } \\
\text { of the } \\
\text { incision } \\
\nabla c m \rrbracket\end{array}$ & $\begin{array}{l}\text { Angle between } \\
\text { the guide wire } \\
\text { and axis of } \\
\text { the proximal } \\
\text { femur in } \\
\text { lateral } \\
\text { fluoroscopic } \\
\text { view } ₫ \text { degree }\end{array}$ & 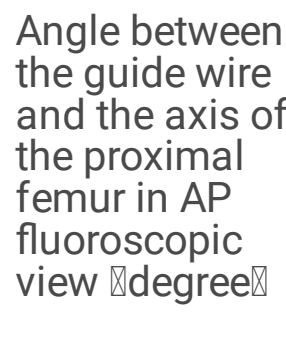 \\
\hline 1 & $M$ & 46 & Left & 1 & 2 & 3 & 5 \\
\hline 2 & $M$ & 92 & Left & 1 & 3 & 2 & 8 \\
\hline 3 & $\mathrm{~F}$ & 82 & Right & 1 & 3 & 3 & 6 \\
\hline 4 & $F$ & 74 & Right & 1 & 2.5 & 0 & 7 \\
\hline 5 & $F$ & 100 & Right & 1 & 3.1 & 0 & 5 \\
\hline 6 & M & 72 & Left & 1 & 2 & 1 & 7 \\
\hline 7 & $M$ & 78 & Left & 1 & 2.2 & 0 & 7 \\
\hline 8 & $M$ & 91 & Right & 1 & 3 & 0 & 7 \\
\hline 9 & $\mathrm{~F}$ & 82 & Left & 1 & 2 & 0 & 6 \\
\hline 10 & $\mathrm{~F}$ & 87 & Right & 2 & 2.3 & 0 & 7 \\
\hline 11 & M & 68 & Left & 1 & 2.6 & 0 & 8 \\
\hline 12 & $F$ & 86 & Right & 2 & 3 & 0 & 8 \\
\hline 13 & $\mathrm{~F}$ & 68 & Left & 1 & 2.8 & 0 & 6 \\
\hline 14 & $F$ & 82 & Right & 1 & 2.8 & 2 & 7 \\
\hline 15 & $\mathrm{~F}$ & 88 & Right & 1 & 2.8 & 0 & 5 \\
\hline 16 & $F$ & 91 & Left & 1 & 2.2 & 2 & 8 \\
\hline 17 & $M$ & 66 & Left & 1 & 2.4 & 0 & 7 \\
\hline 18 & $\mathrm{~F}$ & 80 & Left & 1 & 2.3 & 1 & 5 \\
\hline 19 & M & 65 & Left & 1 & 2.5 & 2 & 6 \\
\hline 20 & $\mathrm{~F}$ & 81 & Left & 1 & 2.4 & 2 & 7 \\
\hline 21 & $\mathrm{~F}$ & 72 & Left & 1 & 2.3 & 2 & 8 \\
\hline 22 & $\mathrm{~F}$ & 68 & Left & 1 & 2 & 0 & 8 \\
\hline 23 & $\mathrm{~F}$ & 80 & Right & 1 & 2.8 & 0 & 7 \\
\hline 24 & $F$ & 82 & Left & 1 & 2.8 & 0 & 8 \\
\hline 25 & $\mathrm{~F}$ & 73 & Right & 1 & 3 & 0 & 8 \\
\hline
\end{tabular}




\begin{tabular}{|c|c|c|c|c|c|c|c|}
\hline 26 & $\mathrm{~F}$ & 95 & Left & 1 & 2.1 & 3 & 6 \\
\hline 27 & $\mathrm{~F}$ & 84 & Left & 1 & 3 & 0 & 5 \\
\hline 28 & $M$ & 47 & Right & 1 & 2.4 & 0 & 5 \\
\hline 29 & $M$ & 93 & Right & 1 & 2.1 & 0 & 6 \\
\hline 30 & $F$ & 81 & Right & 1 & 2 & 0 & 7 \\
\hline 31 & $\mathrm{~F}$ & 65 & Left & 1 & 2.3 & 0 & 6 \\
\hline 32 & $M$ & 83 & Right & 1 & 2.2 & 0 & 7 \\
\hline 33 & $\mathrm{~F}$ & 72 & Left & 1 & 2.1 & 0 & 6 \\
\hline 34 & $\mathrm{~F}$ & 88 & Left & 1 & 2 & 0 & 5 \\
\hline 35 & $\mathrm{~F}$ & 83 & Left & 1 & 2.6 & 1 & 4 \\
\hline 36 & $\mathrm{~F}$ & 83 & Left & 1 & 2.7 & 0 & 6 \\
\hline 37 & $M$ & 90 & Right & 1 & 2.6 & 2 & 5 \\
\hline 38 & $\mathrm{~F}$ & 86 & Right & 1 & 2.4 & 0 & 4 \\
\hline 39 & $\mathrm{~F}$ & 78 & Right & 1 & 2 & 3 & 7 \\
\hline 40 & $M$ & 78 & Left & 1 & 3 & 0 & 6 \\
\hline 41 & $M$ & 73 & Left & 1 & 2.3 & 0 & 5 \\
\hline 42 & $\mathrm{~F}$ & 86 & Left & 1 & 2.1 & 0 & 6 \\
\hline 43 & $\mathrm{~F}$ & 85 & Left & 1 & 2.7 & 3 & 5 \\
\hline 44 & $M$ & 57 & Right & 1 & 2.6 & 1 & 6 \\
\hline 45 & $\mathrm{~F}$ & 72 & Left & 1 & 2.9 & 2 & 5 \\
\hline
\end{tabular}

Figures 


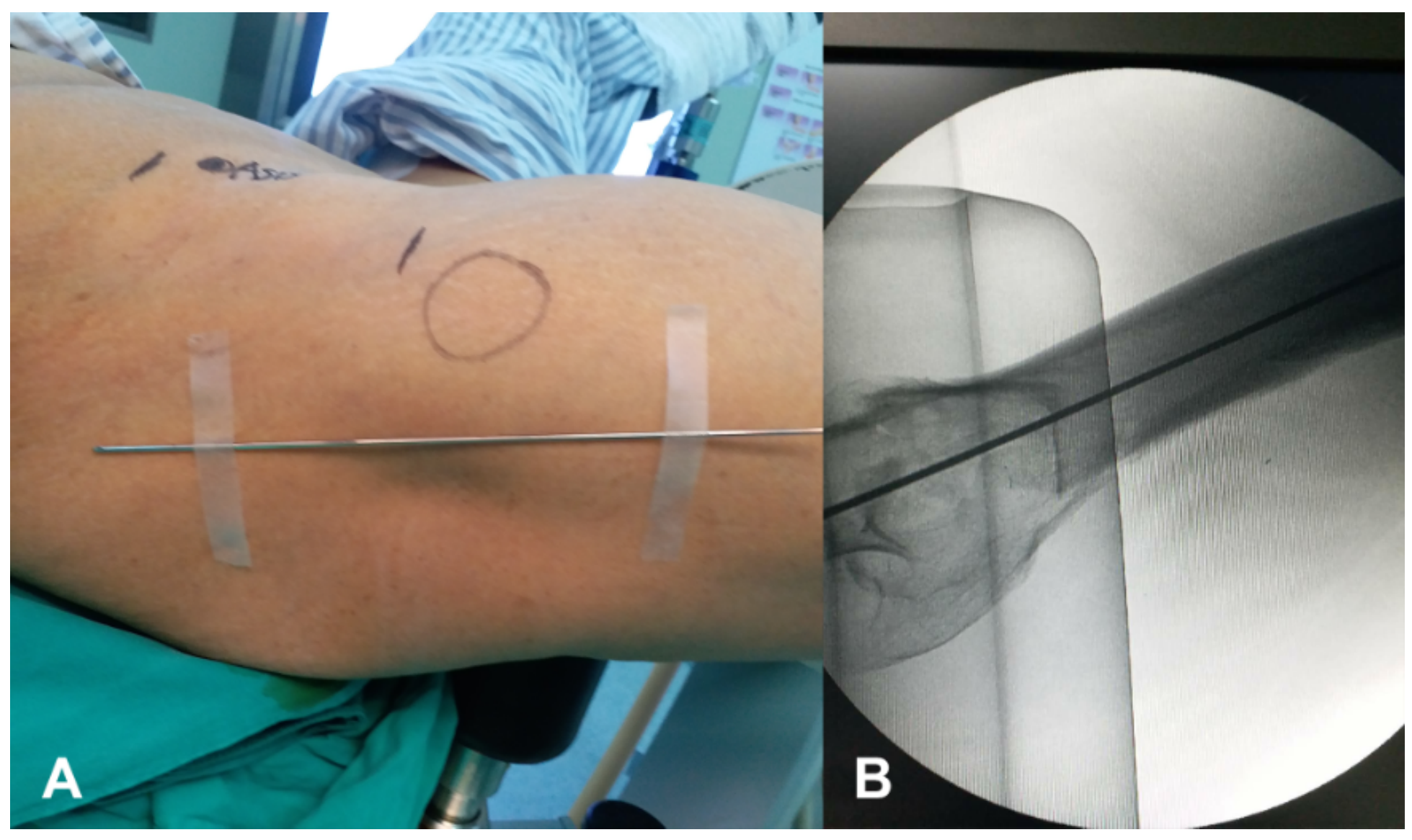

Figure 1

A Kirschner wire was attached to the lateral skin(A); and under lateral fluoroscopy, the projection of the central axis of the proximal femur were determined and marked in the lateral skin $\otimes B \otimes$, 


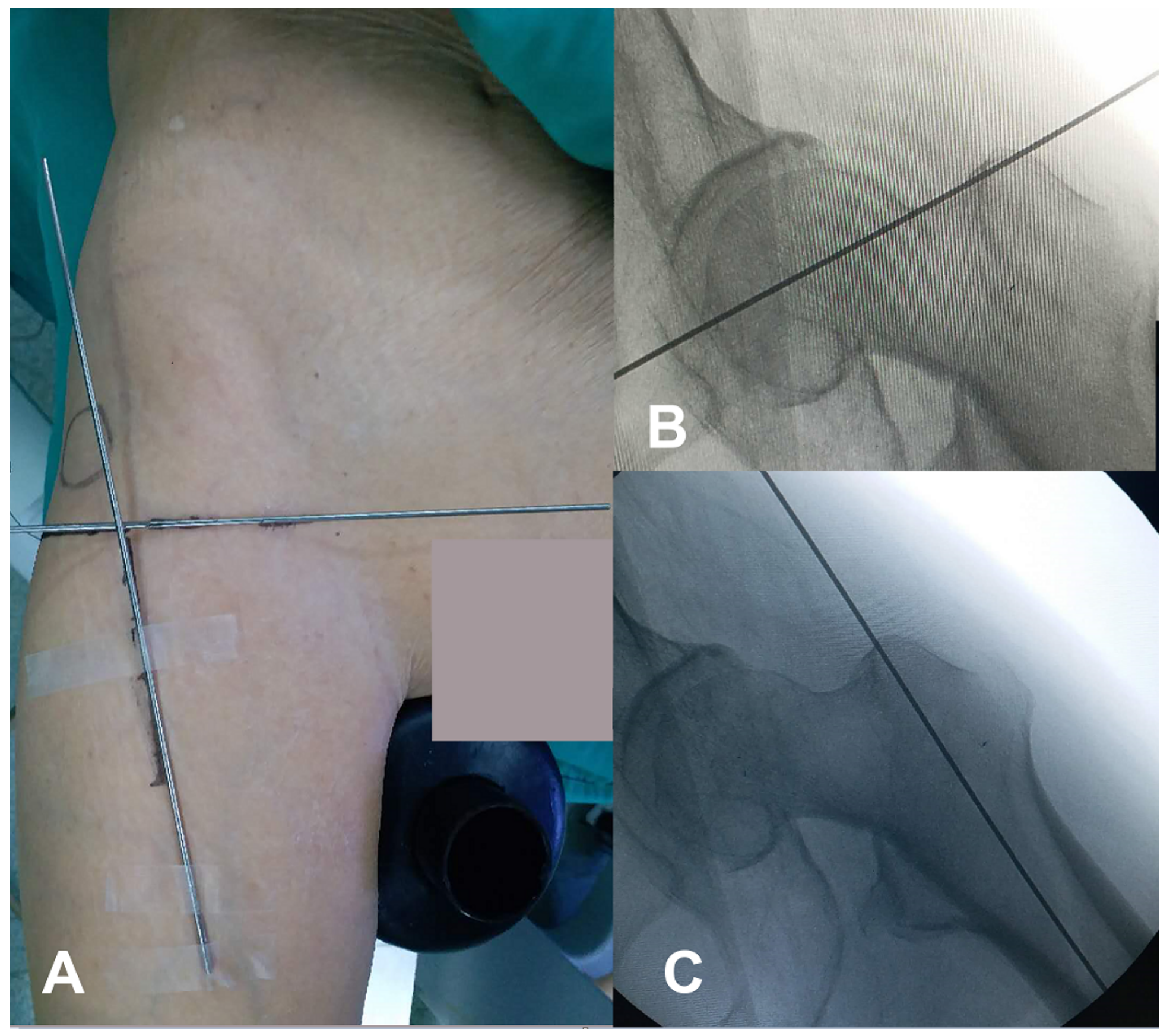

\section{Figure 2}

Two 2-mm-diameter Kirschner wires were placed in the skin over the anterior part of the hip(A). Under A-P fluoroscopy, the first Kirschner wire was placed transversely with its image keeping perpendicular to the longitudinal axis of the femur and running through the tip of the greater trochanter(B) .The second Kirschner wire was placed longitudinal with its image running through the tip of the greater trochanter and being located in the position that the surgeon desired(C). 


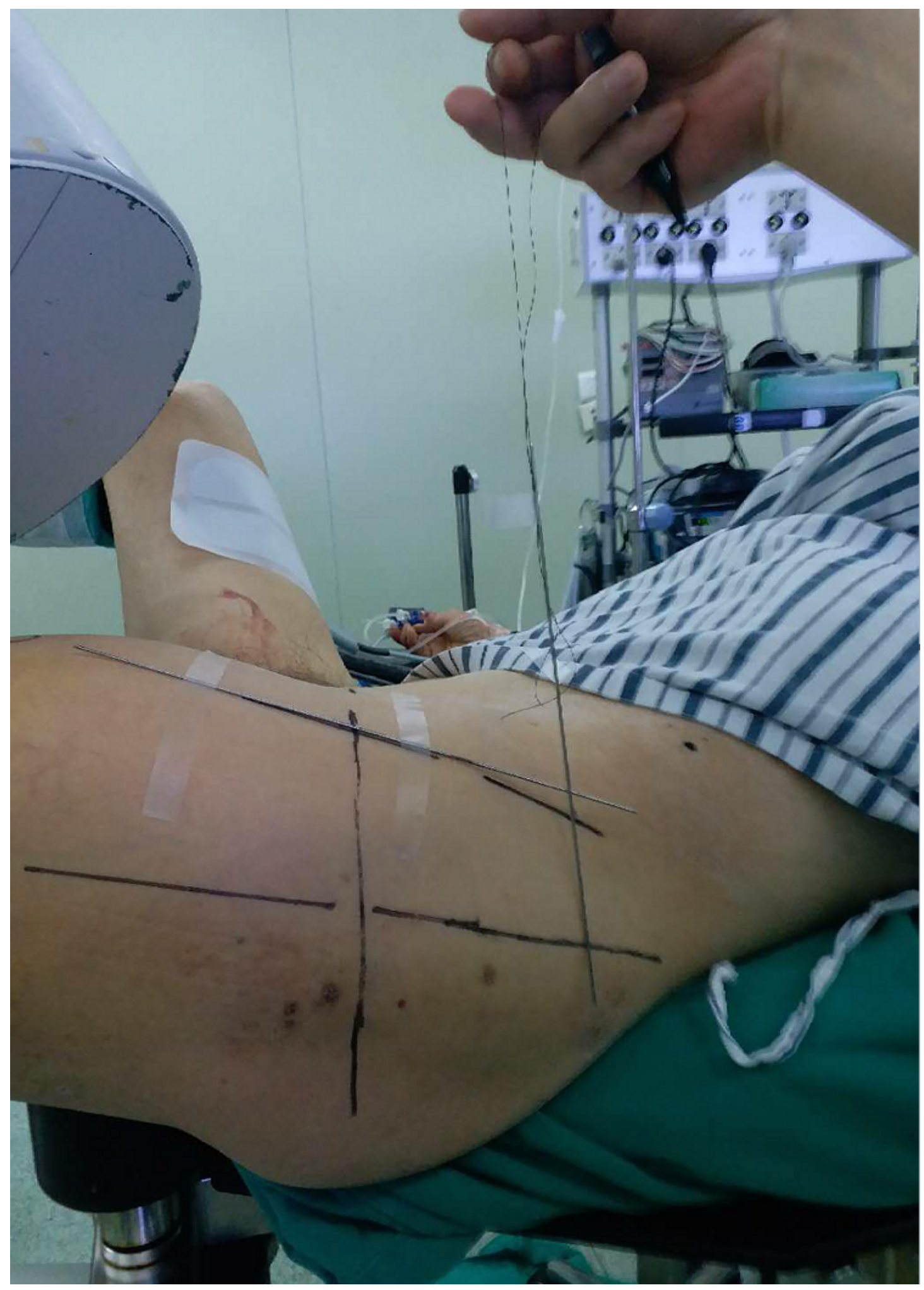

\section{Figure 3}

To determine the entry point of the guide wire in the skin: A 2-mm-diameter Kirschiner wire was suspended and contacted the lateral skin of the thigh and the Kirschner wire that was placed on the anterior part of the hip. The contact point in the lateral skin was marked. A vertical line that ran through this marked point was drawn in the skin. The vertical line crossed with line A in the lateral skin.The crossing point was the entry point of the guide wire in the skin. 


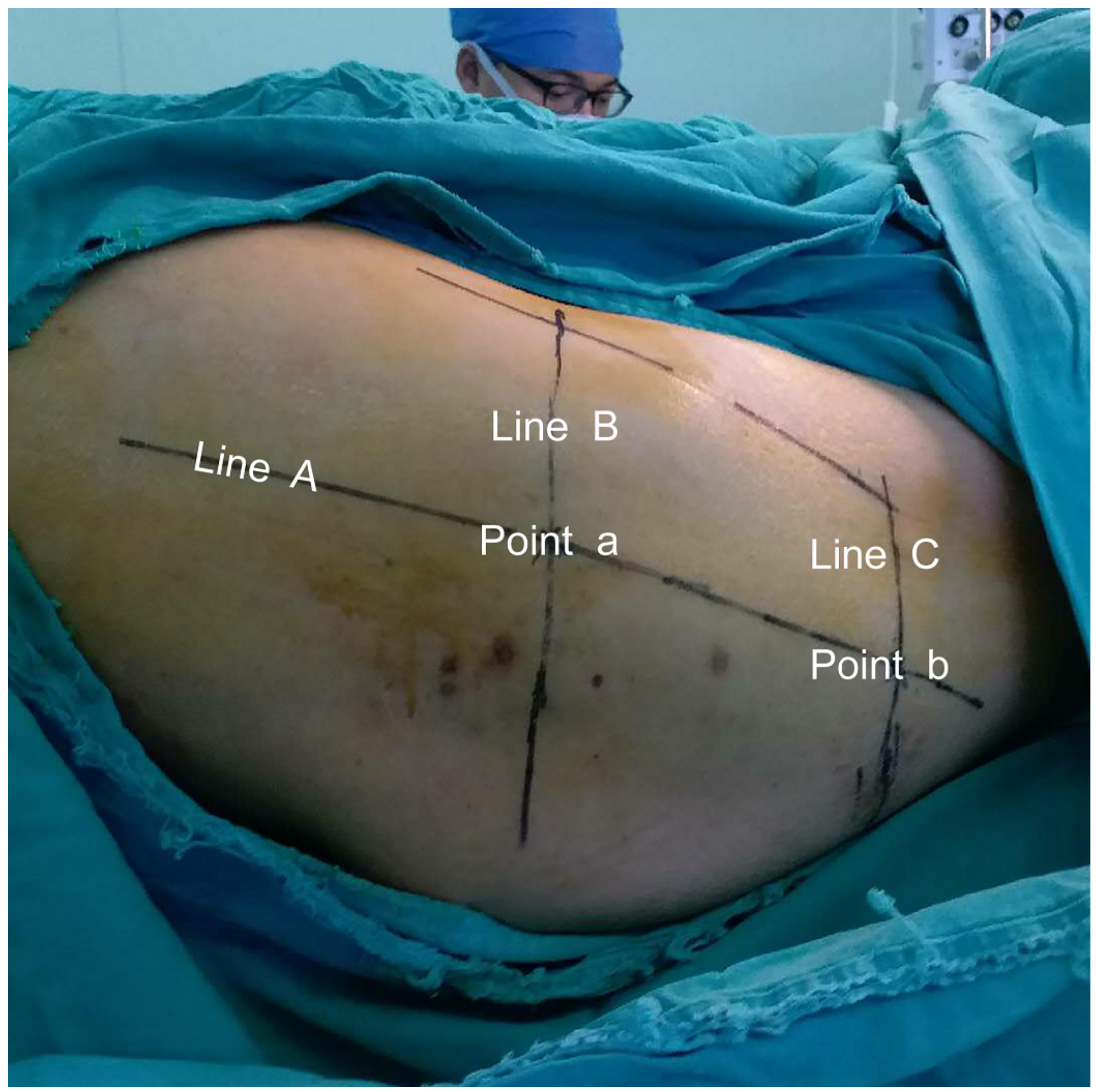

\section{Figure 4}

Line $A$ represents the projection of the central axis of the proximal femur in the lateral skin. Line $B$ (the first vertical line) and Line $C$ (the second vertical line), represent the projected direction of the X-rays beams under A-P fluoroscopy. Point a represents the projection of the tip of the greater trochanter in Line $A$, and the Point $b$ represents the entry point of the guide wire in Line $A$. 


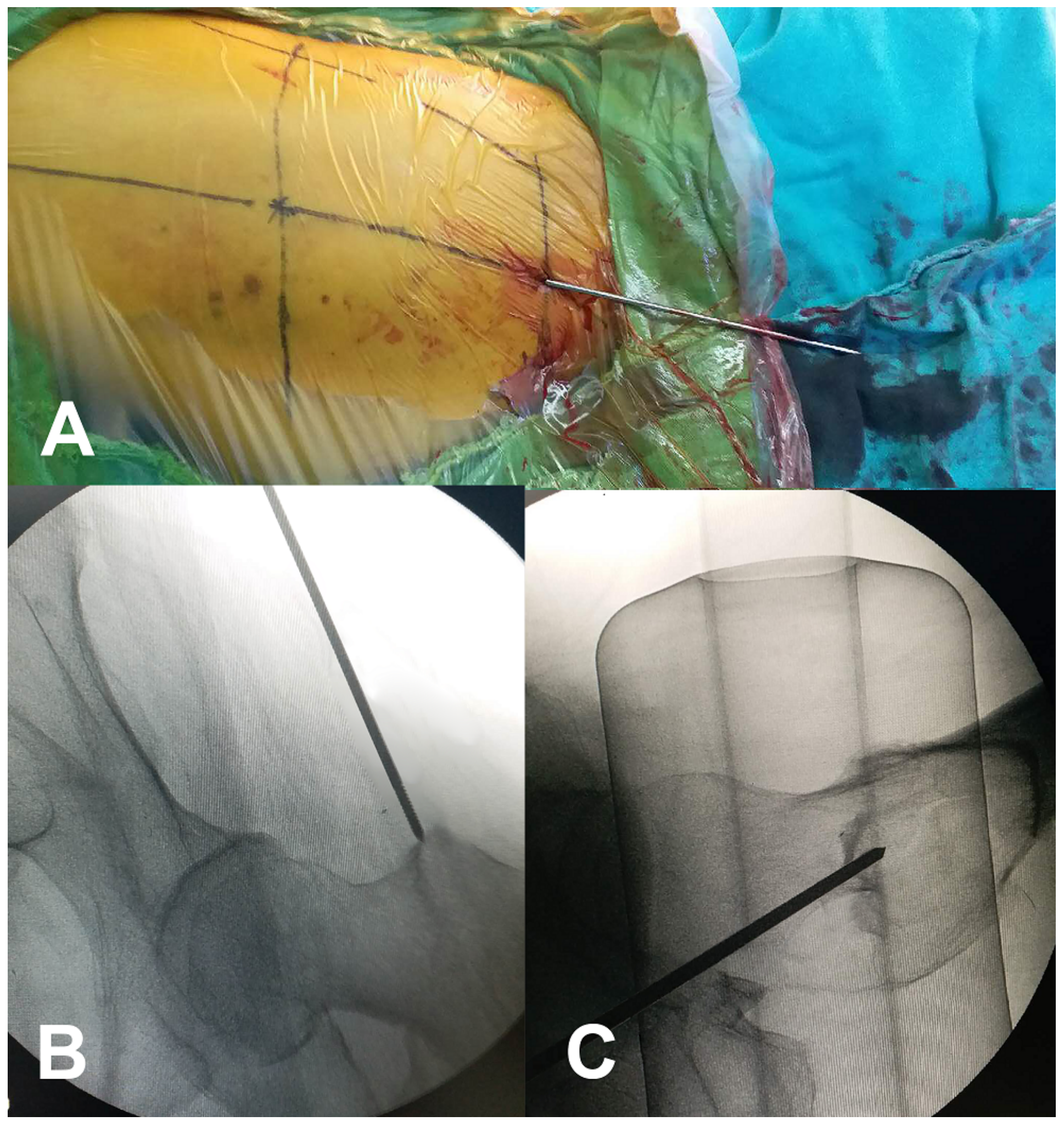

Figure 5

The guide wire was inserted into the hip with its projection at horizontal direction coinciding with Line $A(A)$. Under A-P fluoroscopy, the guide wire run through the tip of the greater trochanter(B). Under lateral fluoroscopy, the image of the guide wire located in the central portion of the proximal femur(C). 


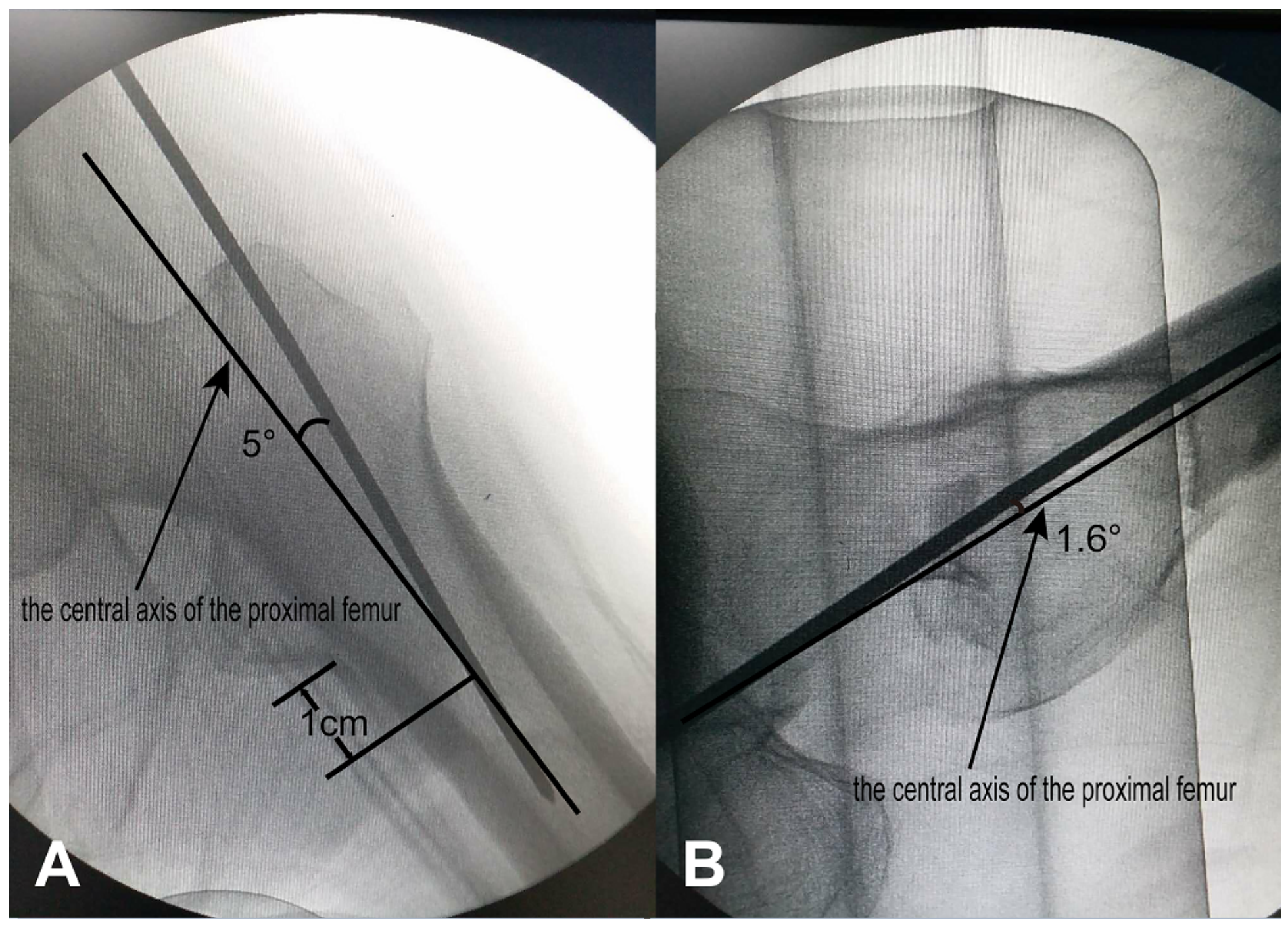

Figure 6

The guide wire was inserted into the bone. Under A-P fluoroscopy, the angle between the guide wire and the axis of the proximal femur should run through the tip of the greater trochanter and the central point of the medullary cavity of this area,which was about $1 \mathrm{~cm}$ away from the inferior edge of the lesser trochanter(A).Under lateral fluoroscopy, the angle between the guide wire and the axis of the proximal femur should be close to $0^{\circ}(B)$. 


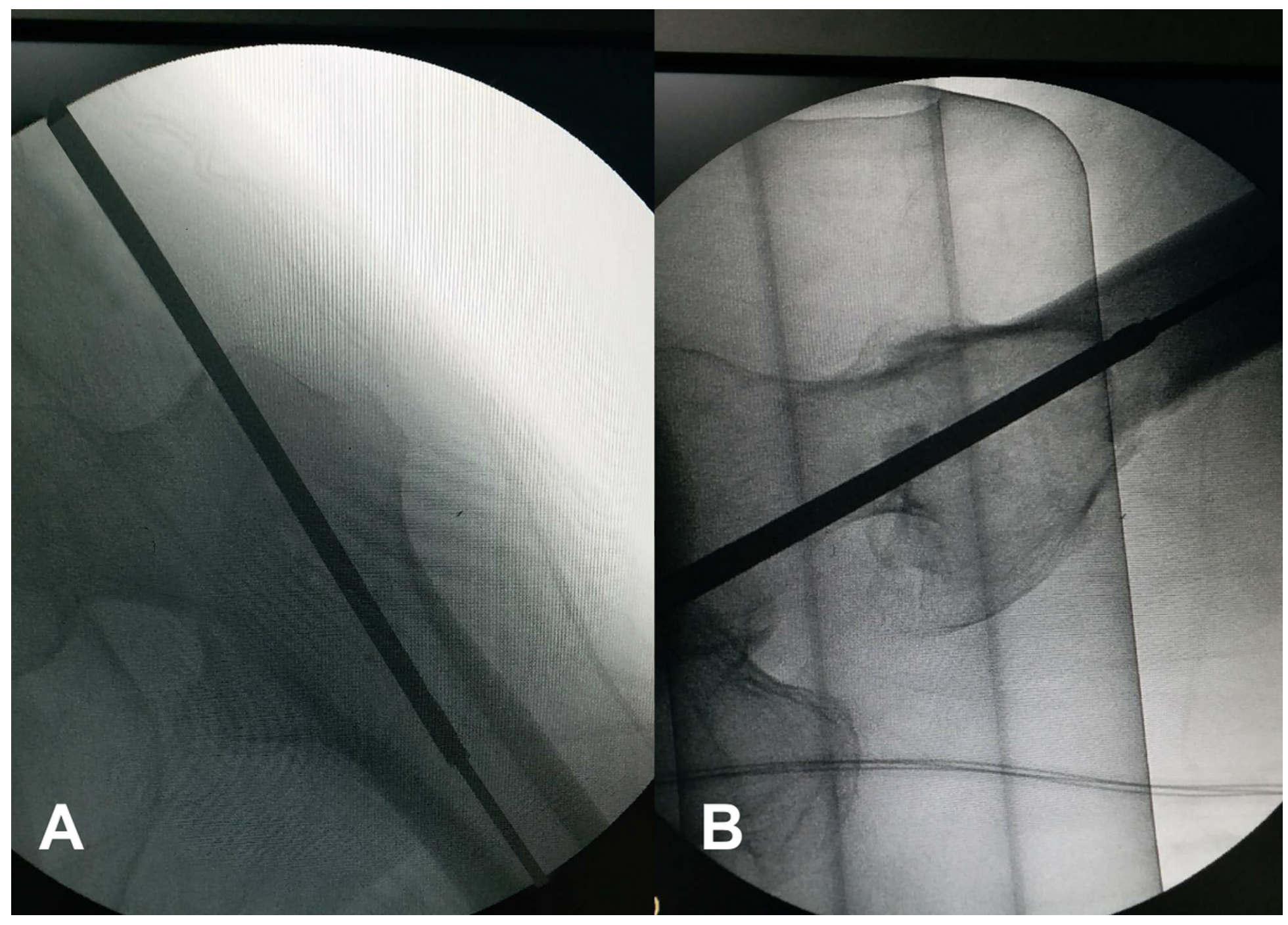

\section{Figure 7}

A 5.5-mm-diameter cannulated drill was used to creat a 5.5-mm-diameter bone canal.Under AP fluoroscopy, the bone canal ran through the tip of the greater trochanter and angled about $5^{\circ}$ with the axis of the proximal femur $\triangle \mathrm{A} \rrbracket$,and under lateral fluorosopic view, it kept parallel to the axis of the proximal femur and located in the central portion of the medullary cavity(B). 


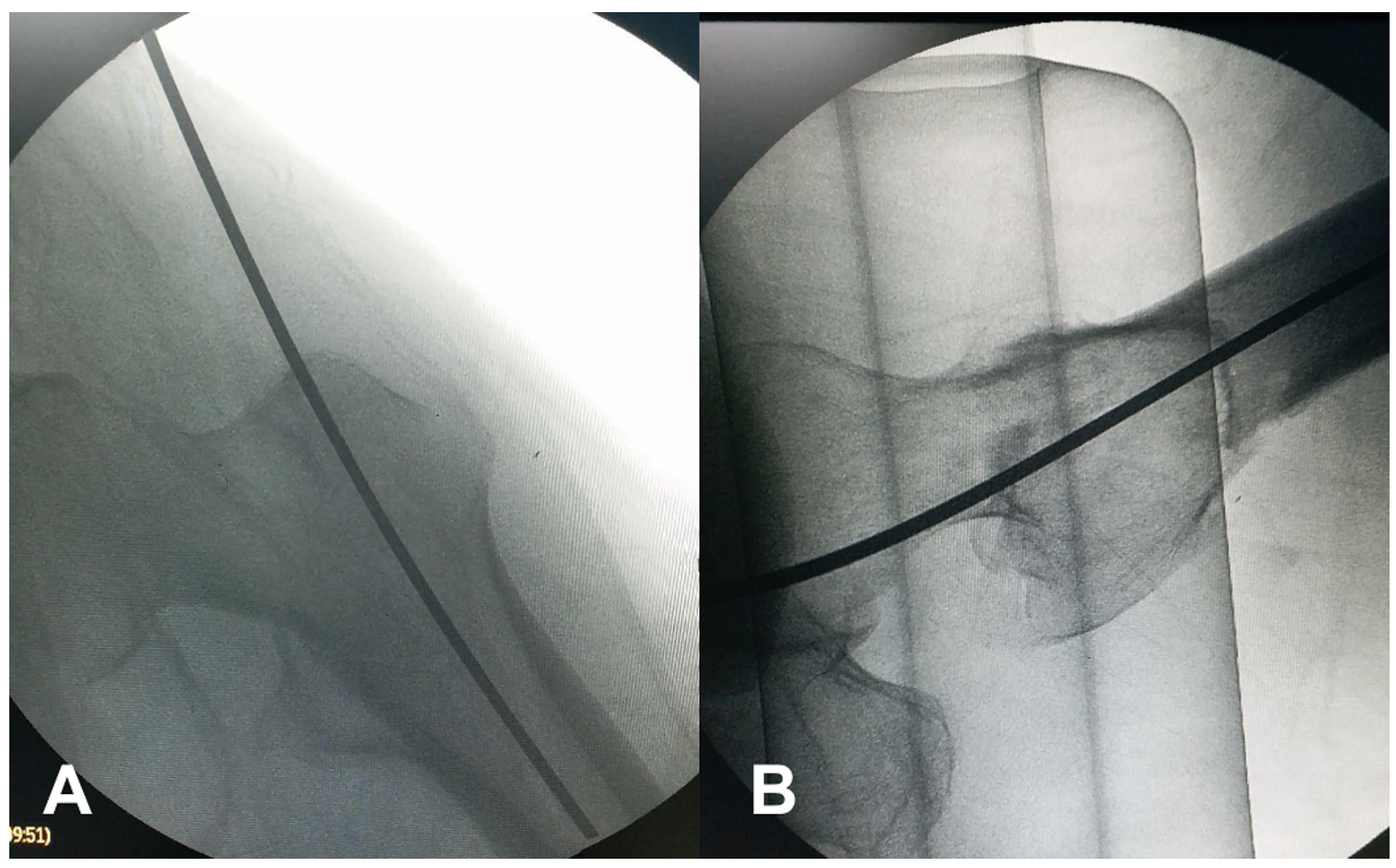

Figure 8

A longer guide wire was inserted into the 5.5-mm-diameter bone canal through another incision to replace the first guide wire. 


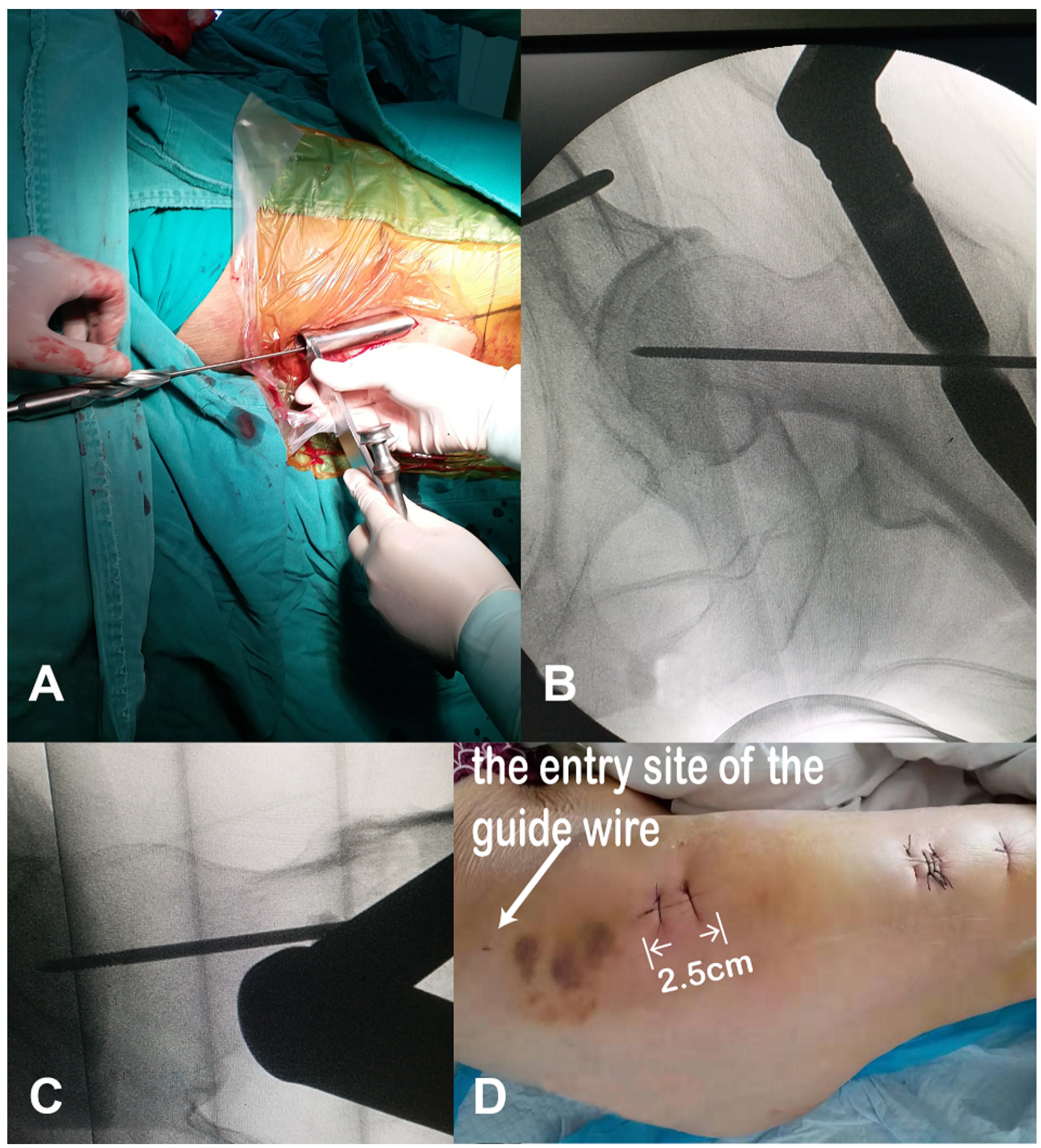

\section{Figure 9}

The cannulated drill was inserted into the soft tissues over the long guide wire to create a bigger bone cananl(A).The A-P (B)and lateral fluoroscopic images(C) of the implant.The length of the incision used for nail insertion was measured and recorded $\triangle \mathrm{D}$ ). 


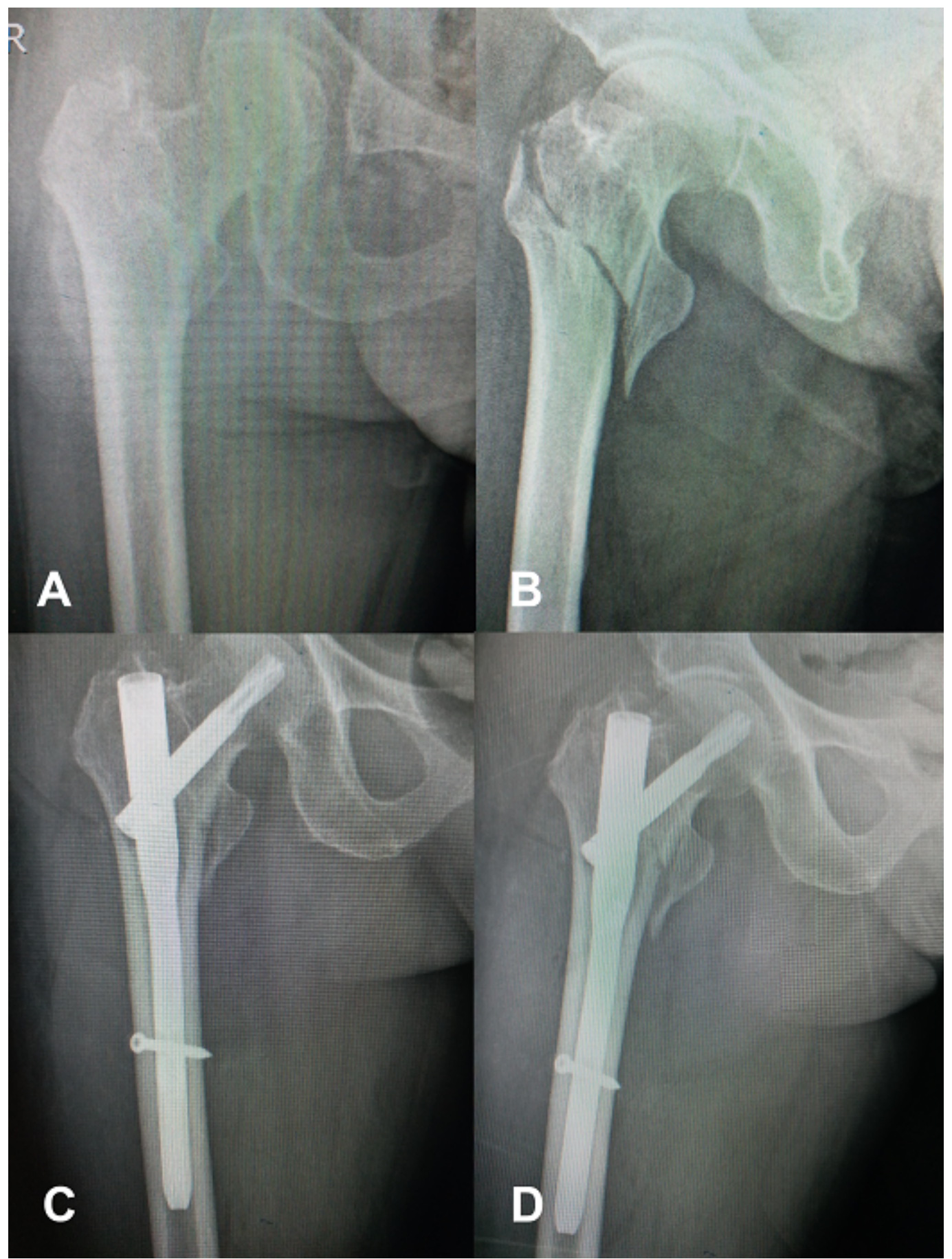

Figure 10

Accurate implant placement. Preoperatve radiographs(A-B)and postoperative radiographs $₫ \mathrm{C}-\mathrm{D} \rrbracket$. 


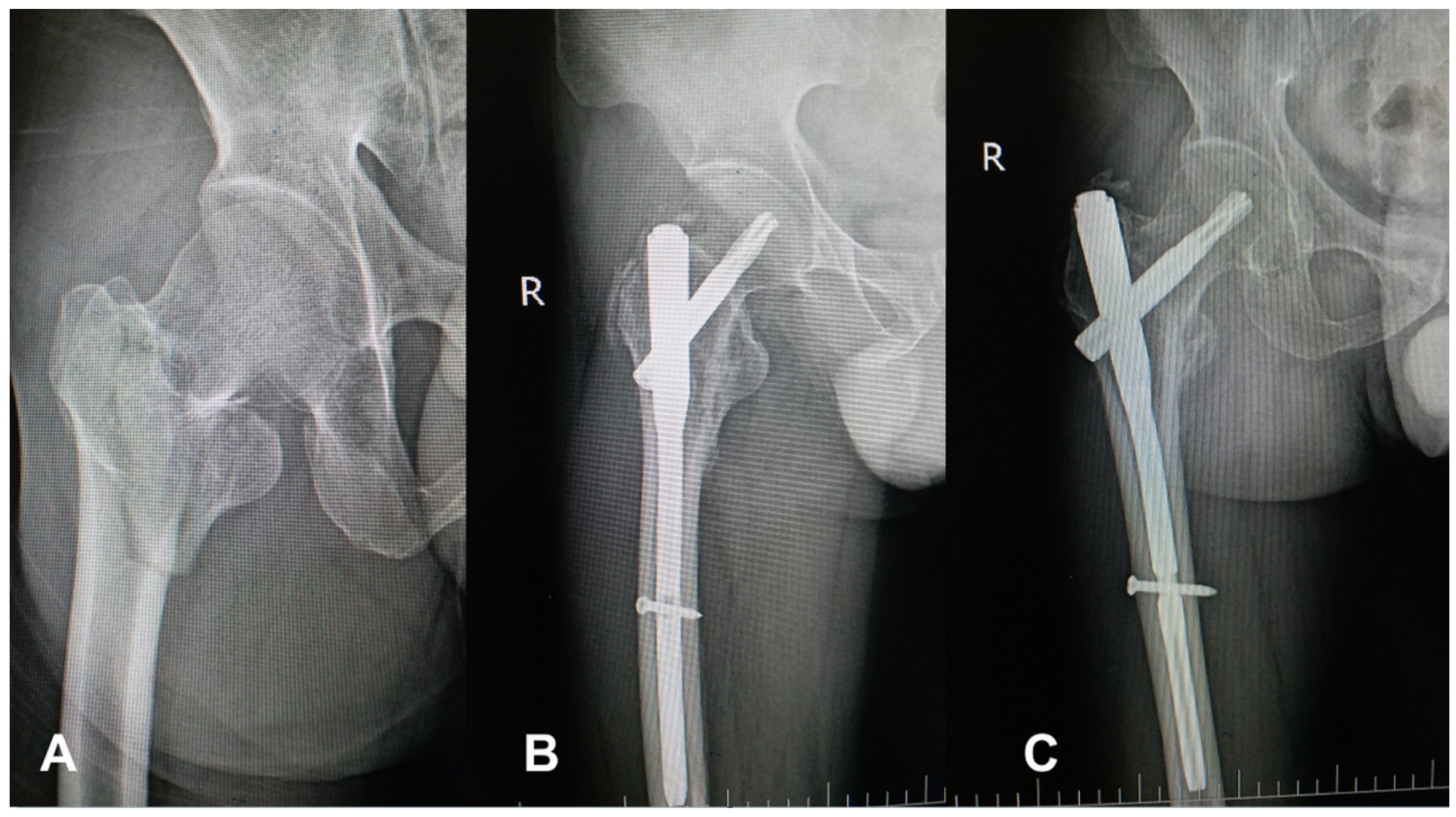

Figure 11

Another patient. Accurate implant placement. Preoperatve radiographs(A)and postoperative radiographs 邓B-C凶.

\section{Supplementary Files}

This is a list of supplementary files associated with this preprint. Click to download.

- renamedfb25f.jpg

- renamedfb25f.jpg

- renamed63215.jpg

- renamed63215.jpg

- renamedb68da.jpg

- renamedb68da.jpg 